Journal of mathematics and computer Science 13 (2014) 353 - 358

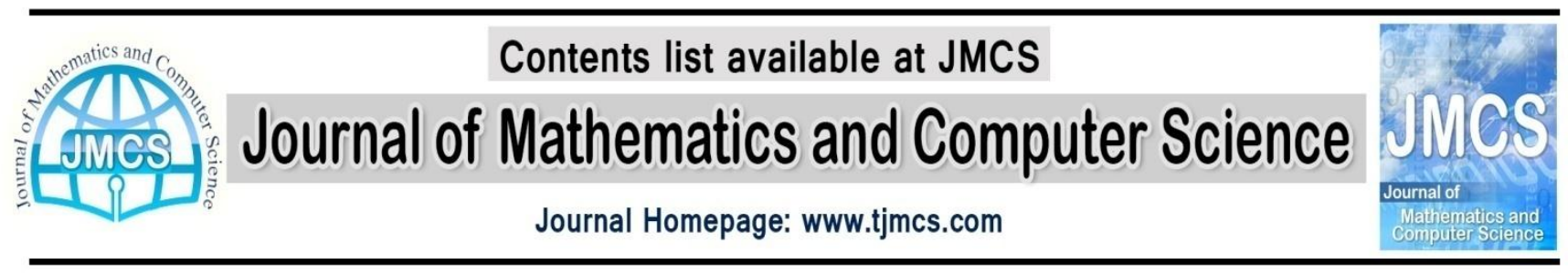

\title{
Proposing a Neural-Genetic System for Optimized Feature Selection Applied in Medical Datasets
}

\author{
Saeed Ayat ${ }^{1}$, Mohammad Reza Mohammadi Khoroushani ${ }^{2, *}$ \\ ${ }^{1}$ Associate Professor, Department of Computer Engineering and Information Technology, \\ Payame Noor University, Iran \\ ${ }^{2}$ M.Sc. student, Department of Computer Engineering and Information Technology, Payame Noor \\ University, Esfahan, Iran \\ 1'dr.ayat@pnu.ac.ir, ${ }^{2}$ mr_mohammadi@of.iut.ac.ir
}

Article history:

Received July 2014

Accepted October 2014

Available online October 2014

\begin{abstract}
This paper, presents a new system for selecting the best optimized features among a collection of features by combination of neural network and genetic algorithm. Feature selection is an important issue because it has a direct impact on the performance (Specificity, sensitivity) and system efficiency.

The proposed system uses neural network for selecting the best features based on Signal to Noise Ratio (SNR), and genetic algorithm for training the neural network by determining the optimum values of weighs and other parameters. This system is a combination of a Multi-Layer Perceptron (MLP) with 3 layers and decimal genetic algorithm

We evaluated our proposed system on 10 medical data sets and compared it with binary genetic algorithm that is used widely for feature selection. The results confirmed the superiority of the proposed system in Specificity, sensitivity and the number of selected optimized features.
\end{abstract}

Keywords: feature selection, optimized feature selection, neural network, genetic algorithm.

\section{Introduction}

Selecting the best optimized features is set among the NP-Hard issues which is it is concerned with optimization. The different methods regarding feature selection issues are of the filter and wrapper categories [1] which have under gone evaluations. Since the wrapper methods, are able to correspond to the learning machine algorithm, they provide better results in comparison with that of the filter methods-In these methods, the growing number of the features of training samples is needed to grow exponentially. In this case, the computational cost increases. So it is very important to find the appropriate method. Since the problem of optimized feature selection is part of optimization problems, evolutionary algorithms like genetic algorithm has mostly been studied and used [2, 3]. In some studies, a combined method is used. They aim to take advantage of algorithms for achieving an 
efficient method respectively [4, 5, 6]. A combined method of neural and genetic algorithm is recommended regarding the feature selection by [7] and [8]. In this study a combined neural-genetic approach is being introduced followed by evaluation of the performance and efficiency of these approaches by comparing them to the binary genetic algorithm regarding effective features selection. In a neural network learning is defined as determining the optimum values of weighs and other parameters like bias and the motion derivative gradient for more efficiency. In network learning the objective is to increase the network efficiency by reducing the errors between real output and network output for the training data and being tested against proper changes in weighs and other parameters of the network. The common approach in this method is the back propagation of the errors. Knowing that learning is a process regarding optimization, it is assumed that the evolutionary algorithms like genetic, Imperialist Competitive Algorithm (ICA), Particle Swarm Optimization (PSO), etc. might be able to increase the efficiency and performance in the neural networks regarding feature selection in its most suitable sense.

In this proposed combined approach the neural network is applied as the most suitable feature selector and the genetic algorithm is applied as a training algorithm. Here, the neural network advantages on the genetic algorithm are combined and the proper balance between efficiency (process speed) and performance (accuracy in pattern Recognition) is found and compared with the studies conducted by $[7,8]$. The proposed combined approach is compared to the binary evolutionary genetic algorithm [9].

\section{Proposed feature selection system}

In this study the signal to Noise ratio, (SNR) criterion, [10] is applied. This criterion makes a feature's weight outstanding in a sense that the weights connected to features of low importance have small values or approaching zero, while the opposite is true which leads to (absolute big value). This approach is of the following two sections. The first section is responsible to select the candid chromosomes (the optimum value of weighs and the biases) to be used in section two. The Fitness function apply the recommended weight to the network, next the determined data set is applied to the network and the mean square of the errors are computed and returned to the genetic algorithm as the value of the Fitness. The error value here, is the result of the dataset outcome and the result if the network outcome, calculated through the mean square error (MSE) equation; hence, the final fitness value which is returned to the system.

The error is the result of the dataset outcome and the network outcome that calculated by the mean square error (MSE) equation. This error returned to the network as fitness feedback.

The second section is responsible to prune the architecture of the candidate chromosome from section one based on (SNR) and categorize the related training samples.

The steps of this approach consist of:

1. The SNR is computed by using the weights that are related to the obtained chromosomes from section1. Then the weights smaller than one will replace by zero. That means the features related to zero weights will remove from future computations.

2. The value of fitness function in this section is computed as the opposite errors categorization

3. The candidate chromosome that meets the fitness function requirements is selected as the most suitable chromosome with its related features 
Table 1. Descriptions of neural networks applied in section 1 and 2

\begin{tabular}{|l|l|l|}
\hline & $\begin{array}{l}\text { Descriptions of neural networks } \\
\text { applied in section 1 }\end{array}$ & $\begin{array}{l}\text { Descriptions of neural networks } \\
\text { applied in section 2 }\end{array}$ \\
\hline $\begin{array}{l}\text { Number of neurons at the } \\
\text { output layer }\end{array}$ & $\begin{array}{l}\text { Number of each dataset } \\
\text { categories }\end{array}$ & Number of each dataset categories \\
\hline $\begin{array}{l}\text { Number of neurons at the } \\
\text { input layer }\end{array}$ & To the number of features & $\begin{array}{l}\text { To the number of non-zero } \\
\text { chromosome genes }\end{array}$ \\
\hline Intermediate layer & 1 & 1 \\
\hline $\begin{array}{l}\text { Number of neurons at the } \\
\text { intermediate layer }\end{array}$ & 10 & 10 \\
\hline First layer function & sigmoid & sigmoid \\
\hline Second layer function & linear & 0.2 \\
\hline training rate & 0.2 & 500 \\
\hline Repeating & 500 & 0.02 \\
\hline $\begin{array}{l}\text { The squares errors } \\
\text { average }\end{array}$ & 0.02 & \\
\hline
\end{tabular}

The number of intermediate layer neurons at both the sections is selected among 3, 5, 7, 10, 15 and 25 indicating that the best average efficiency is 10 .

The decimal generic algorithm description regarding neural network training is presented in Table 2 . These values are selected on experimented basis.

Table 2. The decimal generic algorithm description

\begin{tabular}{|c|c|c|c|c|c|c|c|c|}
\hline $\begin{array}{l}\overrightarrow{0} \\
\stackrel{0}{0} \\
\stackrel{0}{0} \\
\overrightarrow{0}\end{array}$ & 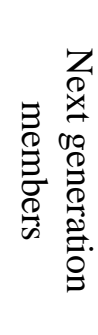 & 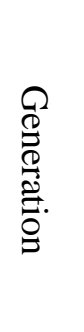 & 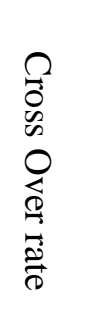 & 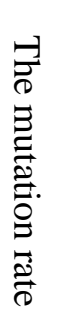 & 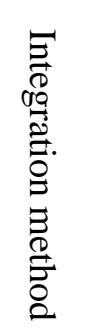 & 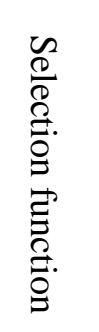 & 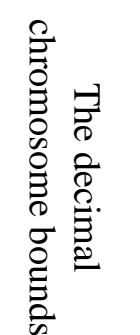 & $\begin{array}{l}\frac{c}{2} \\
\frac{0}{0} \\
\stackrel{0}{0} \\
0 \\
0 \\
\overrightarrow{0} \\
\stackrel{0}{0} . \\
0\end{array}$ \\
\hline 50 & 2 & 100 & 0.08 & 0.2 & $\begin{array}{l}\text { Tow } \\
\text { point }\end{array}$ & Rank & {$[-10,10]$} & $\begin{array}{l}\text { Stochastic } \\
\text { uniform }\end{array}$ \\
\hline
\end{tabular}

The applied definitions and the implemented steps of this logarithm consist of:

a) Coding: beginning the algorithm with a random population of $n$ chromosomes each with a length of 430 features

b) Evaluation: computation of the suitability function according to Table 1

c) Selection: here, two feature vector of (chromosome) are selected through the roulette function and the Fitness average are applied

d) Integration: the selected responses are subject to change. In this approach single point integration is applied where one point is selected along the length of the strip on random basis and the genes (features) are displaced after this point. The Cross Over rate in this study is 0.9.

e) Genetic mutation: this mutation is of 0.005 probability

f) Placing the newborn chromosomes in a collective as the new generation

g) Adding new generation members to the selected members of the initial population of 200 with the new generation 
h) Repetition of the steps beginning from step $b$

The best response is selected after 100 executions.

\section{Evaluation}

The database applied here is from [11] which include 10 data profiles on types of cancer and tumors like brain, kidney, prostate etc. Here $80 \%$ of the samples of each dataset are used in training and $20 \%$ are used for testing.

Table 3.The medical dataset

\begin{tabular}{|l|l|c|c|c|}
\hline Dataset title & Description & $\begin{array}{c}\text { Sample } \\
\text { number }\end{array}$ & $\begin{array}{c}\text { Feature } \\
\text { number }\end{array}$ & $\begin{array}{c}\text { Category } \\
\text { number }\end{array}$ \\
\hline 9 Tumors GEMS & $\begin{array}{l}\text { Nine various human Tumor } \\
\text { types }\end{array}$ & 60 & 5726 & 9 \\
\hline 11 Tumors GEMS & Eleven various human Tumor types & 174 & 12533 & 11 \\
\hline 14 Tumors GEMS & $\begin{array}{l}\text { Fourteen various human Tumor types and 12 } \\
\text { normal tissue types }\end{array}$ & 308 & 15009 & 26 \\
\hline Brain Tumor1 GEMS & Five human brain tumor types & 90 & 5920 & 5 \\
\hline Brain Tumor2 GEMS & Four malignant glioma types & 50 & 10367 & 4 \\
\hline Leukemia1 GEMS & $\begin{array}{l}\text { Acute myelogenous leukemia (AML), acute } \\
\text { lymphoblastic leukemia (ALL) B-cell, and } \\
\text { ALL T-cell }\end{array}$ & 72 & 5327 & 3 \\
\hline Leukemia2 GEMS & $\begin{array}{l}\text { AML, ALL, and mixed-lineage leukemia } \\
\text { (MLL) }\end{array}$ & 72 & 11225 & 3 \\
\hline Lung Cancer GEMS & Four lung cancer types and normal tissues & 203 & 12600 & 5 \\
\hline SRBCT GEMS & Small, round blue cell tumors of children & 83 & 2308 & 4 \\
\hline $\begin{array}{l}\text { Prostate Tumor } \\
\text { GEMS }\end{array}$ & Prostate tumor and normal tissue & 102 & 10905 & 2 \\
\hline
\end{tabular}

\subsection{Evaluating the effectiveness of this proposed approach on feature dimension reduction}

The dimension reduction made through different approaches is presented in Table 4 .

Table 4.Comparison of dimension reduction on medical dataset s through different approaches

\begin{tabular}{|l|c|c|c|}
\hline Dataset title & $\begin{array}{c}\text { Number } \\
\text { of } \\
\text { features }\end{array}$ & $\begin{array}{c}\text { Number of the most } \\
\text { desirable features obtained } \\
\text { through the neural network } \\
\text { using genetic training }\end{array}$ & $\begin{array}{c}\text { Number of the most } \\
\text { desirable features } \\
\text { obtained through binary } \\
\text { Genetic algorithm }\end{array}$ \\
\hline 9 Tumors GEMS & 5726 & 2582 & 2620 \\
\hline 11 Tumors GEMS & 12533 & 4910 & 5345 \\
\hline 14 Tumors GEMS & 15009 & 4845 & 4232 \\
\hline Brain Tumor1 GEMS & 5920 & 635 & 1034 \\
\hline Brain Tumor2 GEMS & 10367 & 995 & 1038 \\
\hline Leukemia1 GEMS & 5327 & 1900 & 1872 \\
\hline Leukemia2 GEMS & 11225 & 633 & 969 \\
\hline Lung Cancer GEMS & 12600 & 3199 & 3211 \\
\hline SRBCT GEMS & 2308 & 264 & 594 \\
\hline Prostate Tumor GEMS & 10905 & 585 & \\
\hline
\end{tabular}




\subsection{Correct classification rate}

The correct classification rate based on two sensitivity and specificities, applied in medical evaluations, are tabulated in Table 5 [12].

Table 5. Correct classification rate of the dataset based on the two sensitivity and specificities

\begin{tabular}{|l|c|c|c|c|}
\hline Approach & \multicolumn{2}{|c|}{$\begin{array}{c}\text { Neural network through genetic } \\
\text { training algorithm }\end{array}$} & \multicolumn{2}{c|}{ Binary Genetic algorithm } \\
\hline & Sensitivity & Specificities & Sensitivity & Specificities \\
\hline 9 Tumors GEMS & 61 & 66 & 56 & 58 \\
\hline 11 Tumors GEMS & 62 & 73 & 62 & 61 \\
\hline 14 Tumors GEMS & 54 & 61 & 50 & 52 \\
\hline Brain Tumor1 GEMS & 66 & 74 & 68 & 73 \\
\hline Brain Tumor2 GEMS & 66 & 76 & 67 & 69 \\
\hline Leukemia1 GEMS & 73 & 66 & 62 & 63 \\
\hline Leukemia2 GEMS & 72 & 76 & 69 & 72 \\
\hline Lung Cancer GEMS & 84 & 91 & 85 & 87 \\
\hline SRBCT GEMS & 58 & 61 & 53 & 55 \\
\hline Prostate Tumor GEMS & 62 & 64 & 61 & 64 \\
\hline
\end{tabular}

The numbers in this table are rounded and presented as percentages. As the performance (Sensitivity, Specificities) evaluation criterion, result of accuracy multiplied by the detecting sensitivity is computed in accordance with [7], since when both these values indicate a rise it means the whole diagnostic accuracy rate has increased. This phenomenon is presented through the following Eqn. :

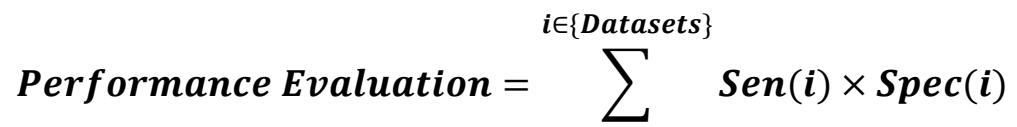

Where, $i$ is one of the 10 dataset $\mathrm{s}$ and $\operatorname{sen}(\mathrm{i})$ and $\operatorname{spec}(\mathrm{i})$ are the diagnostic sensitivity and specificities of the $i^{\text {th }}$ dataset.

\section{Conclusion}

Using neural network in selecting the most desirable features optimized with respect to combined training process, the feature extraction, feature selection and rational classification are of major concern. In this article the signal to noise criterion ratio technique is introduced in selecting the most desirable process through pruning architecture. Training is a process towards optimization and here the meta-heuristic genetic algorithm is applied to select weights with proper and optimized biases in neural network learning algorithm. The results here indicate the establishment of appropriate balance between efficiency and performance. The proposed system here is compared with Genetic Algorithm introduced in other studies like [9] that claimed to be an optimized approach, the evaluation results confirmed the superiority of the proposed system. 


\section{Acknowledgement}

Appreciations are extended to the following esteemed individuals:

- Assistant Prof., Mohammad Reza AkhawanSaraf, FAVA Research Center, Informatics Dept. head and faculty member at Isfahan Industrial University

- $\quad$ MD, SiminHemati, Radiation Treatment Dept. head at Isfahan Medical Sciences University

- MD, Mina Tajvidi, specialist in Dermatology and Radiotherapy Enchology at Seid al Shohada Hospital

- $\quad$ MD, Rezaii specialist in Dermatology and RadioteraphicEnchology at Seid al Shohada Hospital

\section{References}

[1]N. Sanchez-Marono, A. Alonso-Betanzos, M. Tombilla-Sanroman, "Filter methods for feature selection: a comparative study", Proceedings of the 8th international conference on Intelligent data engineering and automated learning, Birmingham, UK, Springer-Verlag: 178-187, 2007.

[2] M. Saberi, D. Safaai, "Feature Selection Method Using Genetic Algorithm For The Classification Of Small and High Dimension Data" in International Journal on IEEE Transaction On Pattern Analysis And Machine Intelligence, VOL. 23, NO. 11, 2005.

[3] M. T. Miller, A. K. Jerebko, J. D. Malley, R. M. Summers, "Feature Selection for Computer-Aided Polyp Detection using Genetic Algorithms", Proceedings of SPIE, Vol. $5031,2003$.

[4] H. Chouaib, O.R. Terrades, S. Tabbone, F. Cloppet, N. Vincent, "Feature selection combining genetic algorithm and Adaboost classifiers" in 19th International Conference on Pattern Recognition(IEEE), ICPR 2008, Tampa, FL,2008.

[5] E. P. Ephzibah, "Cost Effective Approach on Feature selection Using Genetic Algorithms And Fuzzy Logic for Diabetes Diagnosis" , in International Journal on Soft Computing(IJSC), Value 2,No1 , February 2011.

[6] I. S. Oh, J. S. Lee, B. R. Moon, "Hybrid Genetic Algorithms for Feature Selection", IEEE Transaction On Pattern Analysis And Machine Intelligence, VOL. 26, NO. 11, November 2004.

[7]M. Nirooee1, P. Abdolmaleki2, M. Gity, "Designing a Hybrid Model to Differentiate between Malignant and Benign Patterns in Breast Cancer from Mammographic Findings (Text in Persian)", in Iranian Journal of Medical Physics, Pages 67-80, May 2008.

[8] S.Zanganeh, R.Javanmard, M. Ebadzadeh, "A Hybrid Approach for Features Dimension Reduction of Datasets using Hybrid Algorithm Artificial Neural Network and Genetic Algorithm-in Medical Diagnosis" in 4rd Iran Data Mining Conference (IDMC), 2010.

[9] L. Ballerini, X. Li, R. B. Fisher, and J. Rees, "A query-by-example content-based image retrieval system of non-melanoma skin lesions" presented at the Proceedings of the First MICCAI international conference on Medical Content-Based Retrieval for Clinical Decision Support, London, UK, 2010.

[10] A. Verikas. M. Bacauskiene, "Feature selection with neural networks", in Journal Pattern Recognition Letters, Value 23 Issue 11, September 2002 Pages 1323-1335.

[11] gems. (2014, 2014/22/07).Gene Expression Model Selector. Available: http://www.gems-system.org.

[12] M. R. MohammadiKhoroushani, S. Mahzounieh, "Intelligent Skin Cancer Detection Software System based on the principles of telemedicine", in Journal of Hospital, Value 5, Pages 55-60, April 2014. 